\title{
Efficacy and safety of Tibetan medicine Qingpeng ointment for acute gouty arthritis: protocol for a multi-center, randomized, double-blind, placebo- controlled trial
}

\section{Ya-xi Shang}

Beijing University of Chinese Medicine

\section{Xia Dong}

Fangshan Hospital, Beijing University of Chinese Medicine

\section{Zhi-min Xie}

The Second Affiliated Hospital of Zhejiang Chinese Medical University

\section{Xiao-peng Li}

The Second Affiliated Hospital of Zhejiang Chinese Medical University

\section{Xin-chang Wang}

The Second Affiliated Hospital of Zhejiang Chinese Medical University

Ji-yong Huang

The Second Affiliated Hospital of Zhejiang Chinese Medical University

\section{Shu-feng Wei}

Fangshan Hospital, Beijing University of Chinese Medicine

\section{Yuan Liu}

Liuzhou People's Hospital

Jian-ping Liu ( $\square$ jianping_l@hotmail.com )

Beijing University of Chinese Medicine

\section{Research Article}

Keywords: Gout, Acute gouty arthritis, Joint pain, Qingpeng ointment, Tibetan medicine, Randomized controlled trial

Posted Date: September 20th, 2021

DOl: https://doi.org/10.21203/rs.3.rs-708843/v1

License: (9) This work is licensed under a Creative Commons Attribution 4.0 International License. Read Full License 



\section{Efficacy and safety of Tibetan medicine Qingpeng ointment for acute}

gouty arthritis: protocol for a multi-center, randomized, double-blind,

\section{placebo-controlled trial}

Ya-xi Shang ${ }^{1}$, Xia Dong ${ }^{2}$, Zhi-min Xie ${ }^{3}$, Xiao-peng $\mathrm{Li}^{3}$, Xin-chang Wang ${ }^{3}$, Ji-yong

Huang $^{3}$, Shu-feng Wei ${ }^{2}$, Yuan $\mathrm{Liu}^{4}$, Jian-ping Liu ${ }^{1^{*}}$

1 Centre for Evidence-Based Chinese Medicine, Beijing University of Chinese Medicine, Beijing, 100029, China

2 Fangshan Hospital, Beijing University of Chinese Medicine, Beijing 102499, China

3 The Second Affiliated Hospital of Zhejiang Chinese Medical University, Hangzhou 310005, China

4 Liuzhou People's Hospital, Liuzhou 545006, Guangxi Zhuang Autonomous Region, China

Ya-xi Shang,yaxi.s@bucm.edu.cn

Xia Dong, dongxia1222@,163.com

Zhi-min Xie, xzm2019@zjtcm.net

Xiao-peng Li, lixiaopenghz@126.com

Xin-chang Wang, ossani@126.com

Ji-yong Huang, huangjy99@163.com

Shu-feng Wei,weifeng19711009@126.com

Yuan Liu, liuyuanem@163.com

Jian-ping Liu, liujp@bucm.edu.cn

Correspondence to:

Jian-ping Liu, MD, PhD

Professor and director,

Centre for Evidence-Based Chinese Medicine,

Beijing University of Chinese Medicine,

11 Bei San Huan Dong Lu, Chaoyang District,

Beijing, 100029, China

Tel: +86-10-64286760.

E-mail: liujp@bucm.edu.cn 


\begin{abstract}
Background

Acute gouty arthritis (AGA) is an inflammatory arthritis clinically characterized by severe pain, swelling, and restricted movement of joints, which may cause physical disability and decrease quality of life. The use of recommended first-line treatment agents for AGA may be limited by adverse events. There has been a traditional use of alternative therapies for AGA. Tibetan medicine Qingpeng ointment is one of the onmarket herbal products used for symptom relief of AGA. Previous clinical studies indicated that Qingpeng ointment can relieve pain, swelling, redness and dysfunction of joints in patients with AGA. However, there is no rigorous randomized trial to demonstrate its benefit for AGA. In order to evaluate the efficacy and safety of Qingpeng ointment for AGA, we designed a randomized controlled trial.
\end{abstract}

\title{
Methods
}

This study is designed as a multi-center, randomized, double-blind, placebo-controlled trial. Two hundred and six adults with acute flare of gout, and visual analogue scale (VAS) score of joint pain $\geq 3$ points will be recruited. Participants will be randomly assigned to herbal treatment or placebo group at a ratio of 1:1. Qingpeng ointment, or equal placebo ointment will be applied topically at involved joints twice a day for consecutive seven days. Patients in both groups would be allowed giving diclofenac sodium sustained-release tablets as rescue therapy when VAS score of joint pain $\geq 7$ points during the treatment. The primary outcomes will be joint pain measured by VAS score, and joint swelling measured using width and thickness of affected joints and VAS score. Other outcome measures will be joint mobility, joint redness, C-reactive protein, serum uric acid, and the use of rescue medicine as well as adverse effect.

\section{Discussion}

To the best of our knowledge, this study is the first multi-center, randomized, double- 
blind, and placebo-controlled clinical trial to assess the efficacy of Tibetan medicine Qingpeng ointment for AGA. The findings of this study would provide evidence for its use to relieve symptoms of AGA.

\section{Trial registration}

ISRCTN, ISRCTN34355813. Registered 25 January 2021,

https://www.isrctn.com/ISRCTN34355813

\section{Keywords}

Gout, Acute gouty arthritis, Joint pain, Qingpeng ointment, Tibetan medicine, Randomized controlled trial 


\section{Background}

Gout is an inflammatory arthritis occurring in response to monosodium urate crystals formation. It has been recognized as the most common form of inflammatory arthritis [1], with a prevalence of $1.1 \%$ to $3.9 \%$ [2-6]. The disease burden of gout is rising globally [7]. Acute gouty arthritis (AGA) is clinically characterized by severe pain, redness and swelling of joints, and restricted movement, which may cause physical disability, as well as decreased quality of life [8]. Colchicine, nonsteroidal antiinflammatory drugs (NSAIDs) and glucocorticoids are recommended as first-line treatment agents for AGA $[9,10]$. However, comorbidities that may result in contraindications to these drugs are common in patients with gout [11]. Moreover, the use of these drugs may be limited by adverse events. For example, colchicine can increase the rate of diarrhea and gastrointestinal adverse events when compared with placebo and active comparators [12]. NSAIDs are associated with increased risk of gastrointestinal bleeding [13], and may exacerbate renal failure [14]. Glucocorticoids may cause weight gain, cardiovascular disease, diabetes and infections [15]. Therefore, a complementary and alternative therapy that can effectively alleviate the symptoms of AGA with less side effects needs to be found.

Traditional Tibetan medicine (TTM) is a long-established system of traditional medicine with a history of more than 2000 years, and is formed based on the beliefs and practices of the Tibetan culture [16]. TTM consists of numerous botanicals, animal medicines, and mineral medicines, in which many medicines are unique because of the unique growth environment [17]. TTM belongs to traditional minority medicine in China, and plays an important role in the health care system in Tibet Autonomous Region and other Tibetan regions such as Qinghai and Sichuan Provinces in China [16]. Qingpeng ointment is a Tibetan patent medicine for external use, which is convenient and safe to use. Only a few patients who are allergic to the drug may experience skin irritation after using the drug. The main ingredients of Qingpeng ointment are Herba Oxytropis Falcatae (Jidou), Rhei Spiciforme Randix 
(Yadahuang), Radix Aconiti Flavi Et Penduli (Tiebangchui), Chebulae Fructus (Hezi), Terminaliae Belliricae Fructus (Maohezi), Phyllanthi Fructus (Yuganzi), Benzoinum (Anxixiang), Caulis Tinosporae (Kuanjinteng), and artificial Moschus (Shexiang). Qingpeng ointment has the effects of promoting blood circulation, removing blood stasis, reducing swelling and relieving pain, and can be used to treat swelling and pain of joint and muscle caused by rheumatic arthritis, rheumatoid arthritis, osteoarthritis, and gout. Previous clinical studies indicates that Qingpeng ointment can relieve pain, swelling, redness and dysfunction of joints in patients with AGA [18-21]. However, these previous studies have some methodological deficiencies. In order to clearly evaluate the efficacy and safety of Qingpeng ointment for patients with AGA, it is necessary to design and conduct a randomized controlled trial with higher methodological quality.

\section{Objectives}

The aim of this study is to design and conduct a double-blind, randomized, placebocontrolled trial, to evaluate the efficacy and safety of Qingpeng ointment for AGA. The primary objective is to evaluate the efficacy of Qingpeng ointment in relieving the joint pain and reducing the joint swelling of patients with AGA. The secondary objectives are to evaluate the efficacy of Qingpeng ointment in improving the joint mobility and relieving the joint redness in patients with AGA, and to evaluate the safety of Qingpeng ointment.

\section{Methods}

\section{Study design}

This study is a prospective, multi-center, randomized, double-blind, placebo-controlled trial. The study protocol was written in accordance with the Standard Protocol Items: Recommendations for Interventional Trials (SPIRIT) guidelines (Additional file 1 shows the SPIRIT checklist). The trial has been registered on ISRCTN registry: www.isrctn.com. The flowchart of the study design is shown in Fig. 1, and a detailed 
schedule of enrolment, intervention and assessment is shown in Fig. 2.

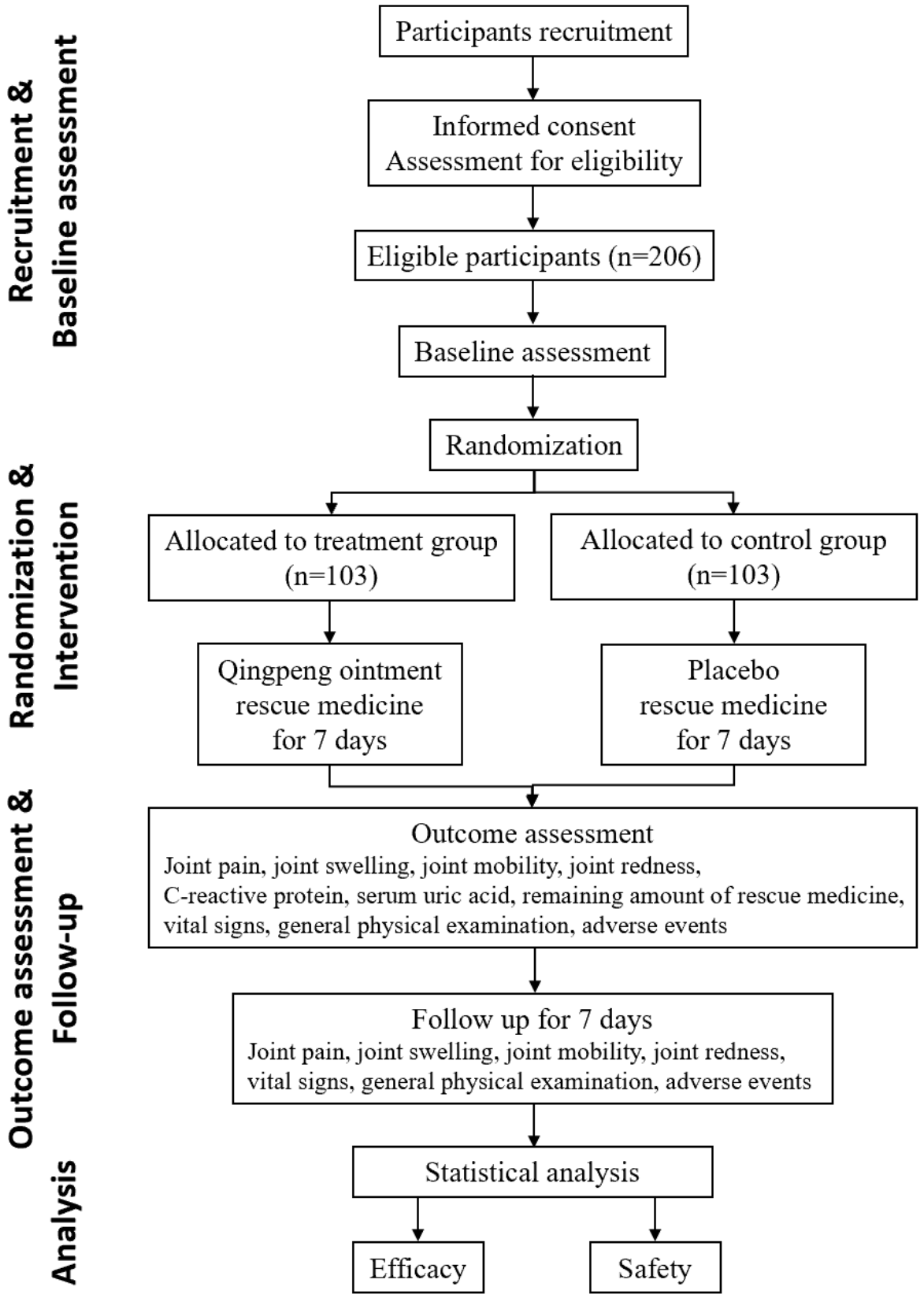

Fig. 1 Trial flow chart 


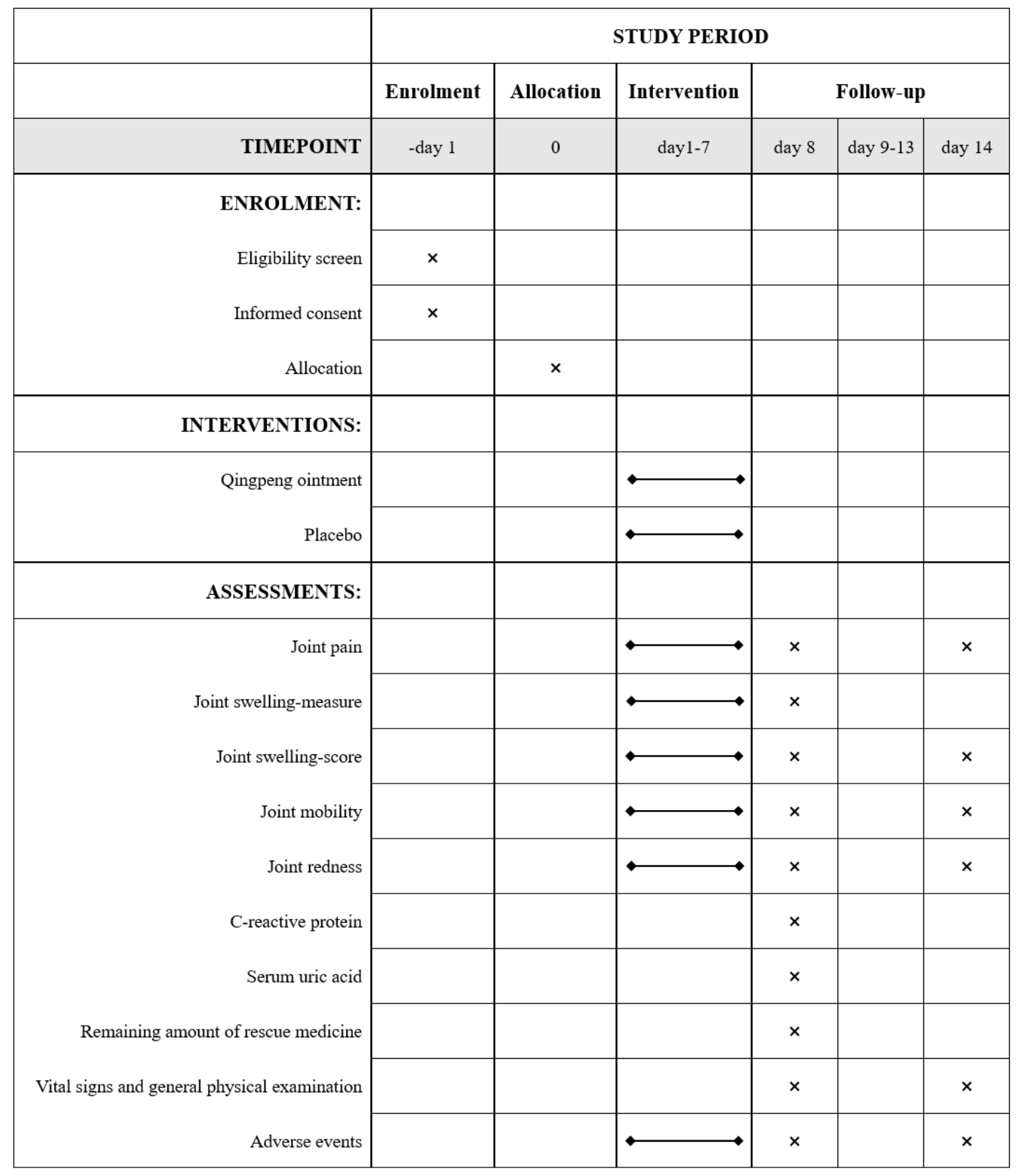

Fig. 2 SPIRIT figure. Schedule of enrolment, interventions, and assessments

\section{Study setting}

This study will be conducted in three hospitals: Fangshan Hospital, Beijing University of Chinese Medicine in Beijing, the Second Affiliated Hospital of Zhejiang Chinese Medical University in Hangzhou, Zhejiang Province, and Liuzhou People's Hospital in Liuzhou, Guangxi Zhuang Autonomous Region.

\section{Study process}




\section{Baseline visit}

After signing an informed consent, patients will be interviewed to obtain information on demographic characteristics, history of past and present illness, and current medications. General physical examination (including height, weight, blood pressure, respiratory rate, heart rate and temperature) and laboratory tests (including serum uric acid and C-reactive protein) will be conducted. Degree of joint pain, swelling, redness and mobility will be scored, and width and thickness of affected joints will be measured. Patients who meet the eligibility criteria will be randomly assigned to treatment group and control group, with 103 patients in each group. Investigators will also instruct participants on how to score the degree of joint pain, swelling, redness and mobility, and how to measure the width and thickness of joints.

\section{Treatment period}

Patients in the treatment group will be treated with Qingpeng ointment, and patients in the control group will be treated with placebo ointment. Patients in both groups will be given Diclofenac Sodium Sustained-Release Tablets (DSSRT) as rescue medicine. Drugs for other underlying diseases such as antihypertensive drugs, hypoglycemic drugs, and hypolipidemic drugs, are permitted to be taken during the treatment period. And other Chinese patent medicine are prohibited during the trial. When participants experience severe skin allergies at application site, or experience serious adverse events, investigators will advise them to discontinue using the study drug. The degree of joint pain, swelling, redness and mobility will be scored every day, and the width and thickness of affected joints will be measured every day. The course of treatment is seven days.

\section{Treatment visit}

After seven days of treatment, participants will return to hospital for treatment visit. General physical examination and laboratory tests (including serum uric acid, Creactive protein) will be conducted. The degree of joint pain, swelling, redness and 
mobility will be scored, and the width and thickness of affected joints will be measured. The combined medication and adverse events during treatment period will be recorded. The remaining study drugs will be recovered, drug distribution and recovery record form will be filled in, and the remaining amount of rescue medicine will be recorded.

\section{Follow-up visit}

Seven days after treatment visit, participants will be followed up. The degree of joint pain, swelling, redness and mobility will be scored, and the adverse events during this seven-days period will be recorded.

\section{Participants and recruitment}

\section{Recruitment and informed consent}

Participants will be recruited from the outpatient department of the listed three hospitals. For all patients who come to the hospital for AGA and are likely to meet the eligibility criteria, investigators will introduce the entire study and informed consent to them, mainly including the study process and intervention methods. Patients will be informed that their participation in this study is entirely voluntary and they are free to withdraw from the study at any time. After signing informed consent and assessed for eligibility criteria, eligible patients will be invited to participate in the study.

\section{Inclusion criteria}

Patients will be eligible for this study if they fulfill the following inclusion criteria: 1. Meeting the ACR (American College of Rheumatology)/EULAR (European League Against Rheumatism) gout classification criteria;

2. Joint pain intensity score (Visual Analogue Scale, VAS, 0-10 points) $\geq 3$ points;

3. With an acute flare of gout, the time from the onset to the visit to hospital should not exceed 1 week;

4. Aged between 18 to 65 years old;

5. Volunteer to participate in the study and sign the informed consent. 


\section{Exclusion criteria}

Patients will not be enrolled into this study if they meet the following exclusion criteria:

1. Also suffering from other types of arthritis;

2. Allergic to the study drugs (Qingpeng Ointment, DSSRT);

3. With severe cardiovascular, cerebrovascular, liver, or kidney diseases;

4. With mental diseases or senile dementia;

5. Women during pregnancy and lactation;

6. With skin ulceration at the affected joint(s);

7. Having newly added uric acid-lowering drugs for any reason in the past week;

8. Participating in other clinical trials at the same time.

\section{Withdrawal or dropout criteria}

Participants will withdraw from this study in the following cases:

1. Deciding to withdraw by themselves;

2. No longer continuing to use the study drugs or accept visits;

3. Having severe allergic reaction at the administration site;

4. Experiencing serious adverse events or complications.

\section{Interventions}

\section{Experimental group}

Participants in treatment group will be treated with Qingpeng ointment (produced by Tibet Cheezheng Tibetan Medicine Co., Ltd; specification: 20g/ointment aluminum tube). Every participant will be given three tubes of ointment. Participants should first clean the skin of affected joints, and then apply the ointment to the skin. The dosage is that the ointment can cover the surface of affected joints, and the thickness is $0.3-0.5$ centimeters. The applied part needs to be massaged gently until the ointment is totally absorbed by the skin. The duration of each application is approximately 0.5 hours, the frequency of application is twice a day, and the interval between two applications is at 
least eight hours. The course of treatment is seven days.

\section{Placebo group}

In order to evaluate the absolute efficacy and safety of Qingpeng ointment for AGA and reduce the risk of bias, we chose placebo as comparison. Participants in control group will be treated with placebo ointment (produced by Tibet Cheezheng Tibetan Medicine Co., Ltd), which is the same as Qingpeng ointment in texture, color, smell and packaging. The usage of placebo ointment is the same as Qingpeng ointment.

\section{Rescue medicine}

DSSRT (produced by Beijing Novartis Pharmaceutical Co., Ltd; specification: $75 \mathrm{mg} /$ tablet, 10 tablets/box) will be given to participants in both treatment group and control group as rescue medicine. Participants in both groups should take the drug only when the VAS score of joint pain is $\geq 7$ points (scale of 0 to 10 ). The recommended dose is one tablet per time, once daily, and the maximum dose is one tablet per time, twice a day.

\section{Assignment of interventions}

\section{Randomization}

Participants will be randomly assigned to treatment group and control group at a ratio of 1:1, with 103 cases in each group. The random sequence was generated by an independent statistician, using the SAS 9.2 software.

\section{Allocation concealment and blinding}

Sequentially numbered drug containers of identical appearance were used to achieve allocation concealment. 206 drug boxes with identical appearance were numbered as 001-206. According to the random sequence, Qingpeng ointment and placebo ointment were put into the corresponding numbered boxes, with three tubes of Qingpeng ointment or placebo ointment in each box. One box of DSSRT was also put into each 
drug box at the same time. Special personnel are assigned to manage the study drugs in each hospital, and the corresponding sequence of drugs will be distributed to each patient according to their recruitment sequence. Throughout the study, the investigators and the participants will not know which group the participants are assigned to. For each participant sequence, a corresponding emergency letter was made, which records the allocation result of that participant. When a participant experience serious adverse events and the allocation result needs to be known, investigators can open the emergency letter. The process of unblinding, including reasons, time, and treatment results, should be recorded on the case report form.

\section{Outcomes}

\section{Primary outcomes}

1. Joint pain: Degree of joint pain will be measured by participants, using a visual analogue scale (VAS, $0-10$ points, $0=$ no pain, $10=$ intolerable pain), at baseline, every day during treatment, and after 7-days treatment.

2. Joint swelling:

2.1 The width and thickness of each affected joint will be measured using vernier calipers (brand: Ruineng, model: NR0139) at baseline, every day during treatment, and after 7-days treatment.

2.2 Degree of joint swelling will be measured by participants, using a visual analogue scale (VAS, $0-10$ points, $0=$ no swelling, $10=$ intolerable swelling), at baseline, every day during treatment, and after 7-days treatment.

\section{Secondary outcomes}

1. Joint mobility: Degree of joint mobility will be measured by participants, using a 0 4 points scale $(0=$ the mobility is normal, and is not restricted; $1=$ the mobility is slightly restricted, but normal activities can still be performed; $2=$ the mobility is moderately restricted, patient is unable to perform general activities, and is able to take care of self daily life; $3=$ the mobility is severely restricted, pain is unbearable when the joint moves, 
patient is unable to take care of self daily life; $4=$ the joint is unable to move), at baseline, every day during treatment, and after 7-days treatment.

2. Joint redness: Degree of joint redness will be measured by participants, using a 0-3 points scale $(0=$ the skin color is normal; $1=$ the skin is slightly red; $2=$ the skin is obviously red; $3=$ the skin is dark red), at baseline, every day during treatment, and after 7-days treatment.

3. C-reactive protein: Will be measured from a blood sample taken at baseline and after 7 days of treatment.

4. Serum uric acid: Will be measured from a blood sample taken at baseline and after 7 days of treatment.

5. Remaining amount of rescue medicine: Will be recorded after 7 days of treatment.

\section{Safety outcomes}

1. Vital signs and general physical examination: Will be tested and recorded at baseline visit, treatment visit and follow-up visit.

2. Adverse events: Adverse events during the trial will be recorded in detail.

\section{Sample size estimation}

This study includes two primary outcomes: joint pain and joint swelling. Sample size was estimated based on these two primary outcome measures. Estimation according to joint pain was based on results from a previous study [20]. In this study, 47\% participants in treatment group (Qingpeng ointment plus diclofenac sodium tablets) had significant relief of joint pain (the score of joint pain degree measured by a selfdeveloped scale reduced by 6 points), and 25\% participants in control group (diclofenac sodium tablets) had significant relief of joint pain. On the basis of a 5\% type I error rate $(\alpha=0.05)$ and a power of $90 \%(\beta=0.1)$, the sample size of each group was 82 . Assuming a 20\% dropout rate, a total of 206 participants should be recruited, with 103 participants in each group.

Estimation according to joint swelling was likewise based on results from another 
previous study [21], which established score of joint swelling measured by a 0-3 points scale as 0.45 points after receiving Qingpeng ointment plus Etoricoxib tablets, and the estimated standard deviation is 0.37 . Score of joint swelling after receiving vaseline plus Etoricoxib tablets is 0.87 , and the estimated standard deviation is 0.64 . On the basis of a $5 \%$ type I error rate $(\alpha=0.05)$ and a power of $90 \%(\beta=0.1)$, the sample size of each group was 27. Assuming a $20 \%$ dropout rate, a total of 68 participants should be recruited, with 34 participants in each group.

Based on these results, we chose the larger sample size of 206 participants, with 103 participants in each group.

\section{Data collection and management}

Data will be collected on case report forms by investigators at baseline visit, treatment visit, and follow-up visit. During treatment period, data will be collected through patient log. Reports of laboratory findings will also be collected and recorded. For participants who withdraw from the trial, the investigator will contact the participants as much as possible to obtain all measurable outcome data. Every investigator should collect and record the data carefully to guarantee its accuracy. Data will then be entered into EpiData manager by two investigators independently, and checked to ensure the accuracy. Personal information of participants will be kept confidential. Every participant will be assigned a participant number, and all data will be identified using this number, instead of directly displaying personal information. All research documents will be saved for at least 5 years after the end of the trial.

\section{Statistical analysis}

Statistical analysis will be performed using SAS 9.2 software, with a statistical significance of $\mathrm{p}$ value less than 0.05 . Normally distributed continuous variables will be described using mean \pm standard deviation (SD), and non-normally distributed continuous variables will be described using median and interquartile range (IQR). Categorical variables will be described using frequencies or percentages. Primary 
outcomes will be analyzed according to the intention-to-treat principle. Intra-group differences of continuous variables will be tested for significance using paired-samples $\mathrm{t}$ test when the data is normally distributed, and will be tested using non-parametric test when the data is non-normally distributed. Between-group differences of continuous variables will be tested for significance using independent-samples t test when the data of the two groups are normally distributed and have equal variance, and will be tested using non-parametric test when the data of the two groups are non-normally distributed or have unequal variances. Chi-square test will be used for categorical data.

\section{Quality control}

Before the start of the study, the investigators will be trained, so that they will have a consistent understanding of the study protocol, and can teach participants how to score the degree of joint pain, swelling, redness and mobility, and how to measure the width and thickness of affected joints. The investigators will also be trained how to fill in the case report form, and they should fill in the form truthfully and completely as required. The original examination report or its copy during the study should be pasted on the case report form. Special personnel will be set up in each center, who will be responsible for the management, distribution, and recovery of the study drugs. The drug distribution and recovery record form will be filled in when distributing and recovering the study drugs. Center for Evidence-Based Chinese Medicine, Beijing University of Chinese Medicine, as the coordinating center of this trial, will assign clinical research associates to regularly conduct monitors at each study center, to monitor whether the investigators comply with the study protocol and fill in the case report forms as required, and whether the data collected is accurate.

\section{Ethics}

This study will be completed in accordance with the ethical principles in the Declaration of Helsinki and the Good Clinical Practice (GCP) Guideline. Beijing University of Chinese Medicine is the sponsor of this trial and will monitor and audit this project. 
The study has been approved by ethics committee of Fangshan Hospital, Beijing University of Chinese Medicine, ethics committee of the Second Affiliated Hospital of Zhejiang Chinese Medical University, and ethics committee of Liuzhou People's Hospital. If the protocol needs to be amended, main investigators of this study will meet together to discuss, communicate and draw conclusions on the revision. The revised protocol will be submitted to the ethics committee of each study center for approval, and an application for revision will also be submitted to the trial registry.

\section{Dissemination policy}

Results of this study will be published in a peer-reviewed journal. Authorship will be granted to authors who make important contributions to the creation of the final publication.

\section{Discussion}

AGA is an inflammatory arthritis characterized by severe pain, swelling, and restricted movement of joints, with rising disease burden. The first-line treatment agents recommended in guidelines for AGA are colchicine, NSAIDs and glucocorticoids, whose use may be limited by contraindications and adverse events [9-11]. In order to avoid adverse effects and to attain better efficacy, some complementary and alternative medicines are used to treat AGA. Qingpeng ointment is a Tibetan patent medicine for external use, which has the effects of promoting blood circulation, removing blood stasis, reducing swelling and relieving pain, and can be used to relieve swelling and pain of joint and muscle caused by arthritis. Qingpeng ointment is convenient and safe to use, and only a few patients who are allergic to the drug may experience skin irritation such as itching, rash and blister after using the drug. Results of several previous clinical trials indicated that, Qingpeng ointment can relieve pain, swelling, redness and dysfunction of joints in patients with AGA [18-21]. These studies were all small-sample, unblind, and based on one medical center, which may lead to bias caused by confounding factors. Only two studies reported using appropriate method for 
randomization $[19,20]$. These studies mainly chose colchicine and NSAIDs (such as diclofenac sodium and etoricoxib) as the intervention for control group. There was no previous double-blind, placebo-controlled trial. In order to evaluate the efficacy and safety of Qingpeng ointment for patients with AGA more clearly, we designed this multi-center, randomized, double-blind, placebo-controlled trial.

To the best of our knowledge, this study is the first multi-center, randomized, doubleblind, and placebo-controlled clinical trial to assess the efficacy of Qingpeng ointment for AGA. In terms of outcome selection, considering from the perspective of patients, we chose joint pain and joint swelling as primary outcomes, and joint mobility, joint redness, remaining amount of rescue medicine, $\mathrm{C}$-reactive protein and serum uric acid as secondary outcomes. Different from previous studies, for the primary outcome of joint swelling, we will use vernier calipers to measure the width and thickness of affected joints, and evaluate the relief of joint swelling using the change of width and thickness. We will also use the visual analogue scale to measure the degree of joint swelling. Measuring joint swelling in these two ways is an innovation different from previous studies. Moreover, the intervention and control of this study are only Qingpeng ointment and placebo ointment. Therefore, in order to improve the compliance of participants, we chose DSSRT as a rescue medicine. Participants in both treatment group and control group can take rescue medicine when the VAS score of joint pain is $\geq 7$ points, to relieve the pain. In this way, the compliance of participants can be improved, and the dropout rate of this study can be reduced. After the treatment period, the remaining amount of rescue medicine will be used as a secondary outcome for evaluating the efficacy of Qingpeng ointment.

The limitation of this study is that, the only intervention and control drug are Qingpeng ointment and placebo ointment. Patients with AGA may experience very severe joint pain. The compliance of participants may be affected, if only externally applied drugs are given. In order to improve the compliance of participants, DSSRT will be used as 
rescue medicine. The investigators will also explain the study protocol and medication requirements to participants detailedly, to ensure that the participants fully understand how to use the drugs. During the treatment period, participants will be instructed regularly to apply the drug on time, and come back to hospital for follow-up.

This study is expected to explicitly evaluate the efficacy and safety of Qingpeng ointment for AGA. If the results prove that Qingpeng ointment can effectively relieve symptoms of AGA, and is safe to use, then the application of Qingpeng ointment in the treatment of AGA can be promoted.

\section{Trial status}

This is protocol version 3.0, dated August 2020. The study is currently actively recruiting eligible participants. Recruitment started on March 2021, and is expected to be completed on December 2021. 


\title{
Supplementary information
}

Additional file 1. SPIRIT 2013 Checklist: Recommended items to address in a clinical trial protocol and related documents.

\begin{abstract}
Abbreviations
AGA: Acute gouty arthritis; VAS: Visual analogue scale; NSAID: Nonsteroidal anti-inflammatory drug; TTM: Traditional Tibetan medicine; DSSRT: Diclofenac Sodium Sustained-Release Tablets; SD: Standard deviation; IQR: Interquartile range; GCP: Good clinical practice
\end{abstract}

\section{Ethics approval and consent to participate}

This study has obtained the ethics approval from the Ethics Committee of Fangshan Hospital, Beijing University of Chinese Medicine (Reference No. FZY LK-2020-015), Ethics Committee of The Second Affiliated Hospital of Zhejiang Chinese Medical University (Reference No. 2020-Y003-IH01), and Ethics Committee of Liuzhou People's Hospital (Reference No. GCP2021-015-01). Before participating, all participants will be fully informed of the trial and sign an informed consent.

\section{Consent for publication}

Not applicable.

\section{Availability of data and materials}

The data-sharing plans for this study are unknown and will be made available based on the contract with the company.

\section{Competing interests}

The authors declare that they have no competing interests.

\section{Funding}

This study is being funded by Tibet Cheezheng Tibetan Medicine Co., Ltd. This funding source had 
no role in the design of this study and will not have any role in the collection, analysis, and interpretation of data.

\section{Author's contributions}

JPL conceived the study and led the protocol development. YXS drafted the manuscript. XD, ZMX, XPL, XCW, JYH, SFW and YL helped to develop the protocol and reviewed this manuscript. All authors read and approved the final manuscript.

\section{Acknowledgement}

Not applicable.

\section{Authors' information}

1 Centre for Evidence-Based Chinese Medicine, Beijing University of Chinese Medicine, Beijing 100029, China.

2 Fangshan Hospital, Beijing University of Chinese Medicine, Beijing 102499, China

3 The Second Affiliated Hospital of Zhejiang Chinese Medical University, Hangzhou 310005, China 4 Liuzhou People's Hospital, Liuzhou 545006, China 


\section{References}

1. Singh JA, Gaffo A: Gout epidemiology and comorbidities. Semin Arthritis Rheum 2020, 50(3s):S11-s 16 .

2. Bardin T, Bouée S, Clerson P, Chalès G, Flipo RM, Lioté F et al: Prevalence of Gout in the Adult Population of France. Arthritis Care Res (Hoboken) 2016, 68(2):261-266.

3. Chen-Xu M, Yokose C, Rai SK, Pillinger MH, Choi HK: Contemporary Prevalence of Gout and Hyperuricemia in the United States and Decadal Trends: The National Health and Nutrition Examination Survey, 2007-2016. Arthritis Rheumatol 2019, 71(6):991-999.

4. Liu R, Han C, Wu D, Xia X, Gu J, Guan H et al: Prevalence of Hyperuricemia and Gout in Mainland China from 2000 to 2014: A Systematic Review and Meta-Analysis. Biomed Res Int 2015, 2015:762820.

5. Rai SK, Aviña-Zubieta JA, McCormick N, De Vera MA, Shojania K, Sayre EC et al: The rising prevalence and incidence of gout in British Columbia, Canada: Population-based trends from 2000 to 2012. Semin Arthritis Rheum 2017, 46(4):451-456.

6. Robinson PC, Taylor WJ, Merriman TR: Systematic review of the prevalence of gout and hyperuricaemia in Australia. Intern Med J 2012, 42(9):997-1007.

7. Xia Y, Wu Q, Wang H, Zhang S, Jiang Y, Gong T et al: Global, regional and national burden of gout, 1990-2017: a systematic analysis of the Global Burden of Disease Study. Rheumatology (Oxford) 2020, 59(7):1529-1538.

8. Chandratre P, Roddy E, Clarson L, Richardson J, Hider SL, Mallen CD: Health-related quality of life in gout: a systematic review. Rheumatology (Oxford) 2013, 52(11):2031-2040.

9. FitzGerald JD, Dalbeth N, Mikuls T, Brignardello-Petersen R, Guyatt G, Abeles AM et al: 2020 American College of Rheumatology Guideline for the Management of Gout. Arthritis Care Res (Hoboken) 2020, 72(6):744-760.

10. Richette P, Doherty M, Pascual E, Barskova V, Becce F, Castañeda-Sanabria J et al: 2016 updated EULAR evidence-based recommendations for the management of gout. Ann Rheum Dis 2017, 76(1):29-42.

11. Keenan RT, O'Brien WR, Lee KH, Crittenden DB, Fisher MC, Goldfarb DS et al: Prevalence of contraindications and prescription of pharmacologic therapies for gout. Am J Med 2011, 
124(2):155-163.

12. Stewart S, Yang KCK, Atkins K, Dalbeth N, Robinson PC: Adverse events during oral colchicine use: a systematic review and meta-analysis of randomised controlled trials. Arthritis Res Ther 2020, 22(1):28.

13. Lanas Á, Carrera-Lasfuentes P, Arguedas Y, García S, Bujanda L, Calvet X et al: Risk of upper and lower gastrointestinal bleeding in patients taking nonsteroidal anti-inflammatory drugs, antiplatelet agents, or anticoagulants. Clin Gastroenterol Hepatol 2015, 13(5):906912.e902.

14. Cheng HF, Harris RC: Renal effects of non-steroidal anti-inflammatory drugs and selective cyclooxygenase-2 inhibitors. Curr Pharm Des 2005, 11(14):1795-1804.

15. Costello R, Patel R, Humphreys J, McBeth J, Dixon WG: Patient perceptions of glucocorticoid side effects: a cross-sectional survey of users in an online health community. BMJ Open 2017, 7(4):e014603.

16. Luo H: Traditional Tibetan medicine in China: an overview of current status and prospects. Chin J Integr Med 2015, 21(1):67-70.

17. Jia MR, Zhang Y, Du J: Characteristics and sustainable development of Tibetan medicine. Chin Tradit Herb Drugs 2010, 41(2):326-329.

18. Liu YL: Clinical observation on the treatment of acute gouty arthritis by external application of Qingpeng ointment. Hebei Med J 2011, 33(5):774-775.

19. Luo QN, Wu MQ: Contrastive study of the Qizheng Qing Peng ointment in the treatment of gouty arthritis. China Prac Med 2011, 6(22):19-20.

20. Wang JB, Xie RA, Jiang XB, Pan L, Liang HD: Clinical investigation on the treatment of acute gouty arthritis with Qingpeng paste. Chin J Orthopa Trauma 2006, 19(12):755-756.

21. Yu CZ, Xu XM, Liu X, Yan Z: Observation on the efficacy of Qingpeng ointment combined with etoricoxib in the treatment of gouty arthritis. Mod J Integr Tradit Chin and West Med 2011, 20(33):4217-4218. 


\section{Supplementary Files}

This is a list of supplementary files associated with this preprint. Click to download.

- SPIRITchecklist.pdf 